\title{
CHARACTERIZATION OF DELAY-INDEPENDENT STABILITY AND DELAY INTERFERENCE PHENOMENA
}

\author{
WIM MICHIELS* AND SILVIU-IULIAN NICULESCU ${ }^{\dagger}$
}

\begin{abstract}
The problem of the asymptotic stability independent of delays for a class of linear systems including multiple delays is addressed. Both cases where the delays are allowed to vary independently of each other, and where they are restricted to a one-dimensional subspace of the delay-parameter space are considered. It the latter case it turns out that the resulting dependency between the delays (rationally independent, rationally dependent, commensurate) plays an important role. The stability conditions are expressed in terms of the spectral properties of some appropriate complex matrices. As a consequence of the stability study, a complete characterization of the delay interference phenomenon is given. Furthermore, a connection is established with the stability theory for continuous-time delay-difference equations, subjected to delay perturbations. Various illustrative examples complete the paper.
\end{abstract}

Key words. time-delay systems, stability, delay interference.

AMS subject classifications. 34K06,34K20

1. Introduction. We address the asymptotic stability in the delay-parameter space of the linear system

$$
\dot{x}(t)=A_{0} x(t)+\sum_{i=1}^{m} A_{i} x\left(t-\tau_{i}\right),
$$

where $A_{i} \in \mathbb{R}^{n \times n}, i=0, \ldots, m$, are given system matrices and $\tau_{i} \geq 0, i=1, \ldots, m$, are delay constants.

The subject of delay-independent stability of the zero solution of (1.1), that is, asymptotic stability guaranteed for all delay values, received a lot of attention in the literature starting with the $80 \mathrm{~s}$. Without being exhaustive, we cite some of the approaches proposed to handle the problem: two-variable criteria $[12,13]$, matrix pencil techniques $[3,20]$, frequency-sweeping tests $[5,4]$, and finite-dimensional LMI conditions derived by using some appropriate quadratic Lyapunov-Krasovskii functionals [2]. For further discussions and references, see, for instance, [24, 8, 21, 6]. Essentially, in most of such existing criteria for delay-independent stability and, more generally, in work on characterizing and computing stability regions in the delayparameter space, the delays are either allowed to vary completely independently of each other, or they are restricted to be commensurate (multiples of the same number). The latter implies a relation of the form

$$
\left(\tau_{1}, \ldots, \tau_{m}\right)=\tau_{0}\left(n_{1}, \ldots, n_{m}\right), \quad n_{i} \in \mathbb{N}, i=1, \ldots, m
$$

where $n_{1}, \ldots, n_{m}$ are fixed and $\tau_{0} \in \mathbb{R}_{+}$is the free parameter. Geometrically, the parameterization (1.2) corresponds to a particular ray or direction in the complete delay-parameter space.

In the paper we study the eigenvalue distribution of a collection of complex matrices, derived from the delay system (1.1). Among others, this leads us to new necessary

\footnotetext{
*Department of Computer Science, Katholieke Universiteit Leuven, Celestijnenlaan 200A, 3001 Heverlee, Belgium, tel.+32.16.327537, fax +32.16.327996 (Wim.Michiels@cs.kuleuven.be).

†Université de Technologie de Compiègne, Centre de Recherches de Royallieu, BP 20529 Compiègne, France, tel. +33.3.44234484, fax +33.3.44234477 (Silviu.Niculescu@hds.utc.fr).
} 
and sufficient conditions for delay-independent stability. The criteria cover both the case where all delays vary independently of each other, and where they are restricted to an arbitrary ray in the delay-parameter space, more precisely,

$$
\left(\tau_{1}, \ldots, \tau_{m}\right)=\tau_{0}\left(r_{1}, \ldots, r_{m}\right), \quad r_{i} \in \mathbb{R}_{+}, i=1, \ldots, m
$$

with $\tau_{0} \in \mathbb{R}_{+}$being once again the free parameter. The relaxation from (1.2) to (1.3) has the following consequences:

1. it allows us to make assertions about delay-independent stability when the ray under consideration consists of delay values with any type of interdependence (commensurate, rationally (in)dependent delays). This interdependency turns out to be important;

2. a study of the sensitivity of delay-independent stability along a ray w.r.t. changes of the direction (determined by the $r_{i}$ in (1.3)) becomes possible. Among others, it leads us to a complete characterization of the so-called delay interference phenomenon, that is, the presence of delay-independent stability along a particular ray, which is not robust against arbitrarily small perturbations of the direction of the ray.

Due to the fragility of delay-independent stability properties (note that they involve a non-compact set of delay values), these issues are nontrivial and deserve special attention, as we shall see. They have, however, barely been treated in the literature, excepting some contributions on the interference phenomenon and related topics [16, $17,5,15,22]$.

To the best of the authors' knowledge, the notion of delay interference ${ }^{1}$ was first mentioned by MacDonald [17] (see also [16]), where a second-order system including two delays is shown to be subjected to delay interference if it has the following property: delay-independent stability if the delays are equal, and delay-dependent stability with respect to each delay if the other is equal to zero. A further example (scalar system including two delays) of delay interference can be found in [5]. This is also discussed in [15] and [22], where some characterizations regarding interference are given (a frequency-sweeping test combined with a matrix pencil condition in [22], a sector characterization in [15]). As we shall discuss at the end of Section 5, these results appear as corollaries of the general theory developed throughout the paper.

The remaining part of the paper is organized as follows: some definitions, notations and assumptions are presented in the next section. Section 3 contains preliminary results, in support of Section 4, which is devoted to the main results (delayindependent stability and interference characterizations). In Section 5 some illustrative examples are presented and discussed. In Section 6 the main results are connected with the stability theory of continuous-time delay-difference equations. Finally, some concluding remarks end the paper. The appendices contain some background material.

\footnotetext{
${ }^{1}$ In Physics, interference represents the combination of two or more wave motions to form a resultant wave in which the displacement is reinforced or cancelled.
} 


\section{Notation, definitions and assumptions.}

The following notations are used:
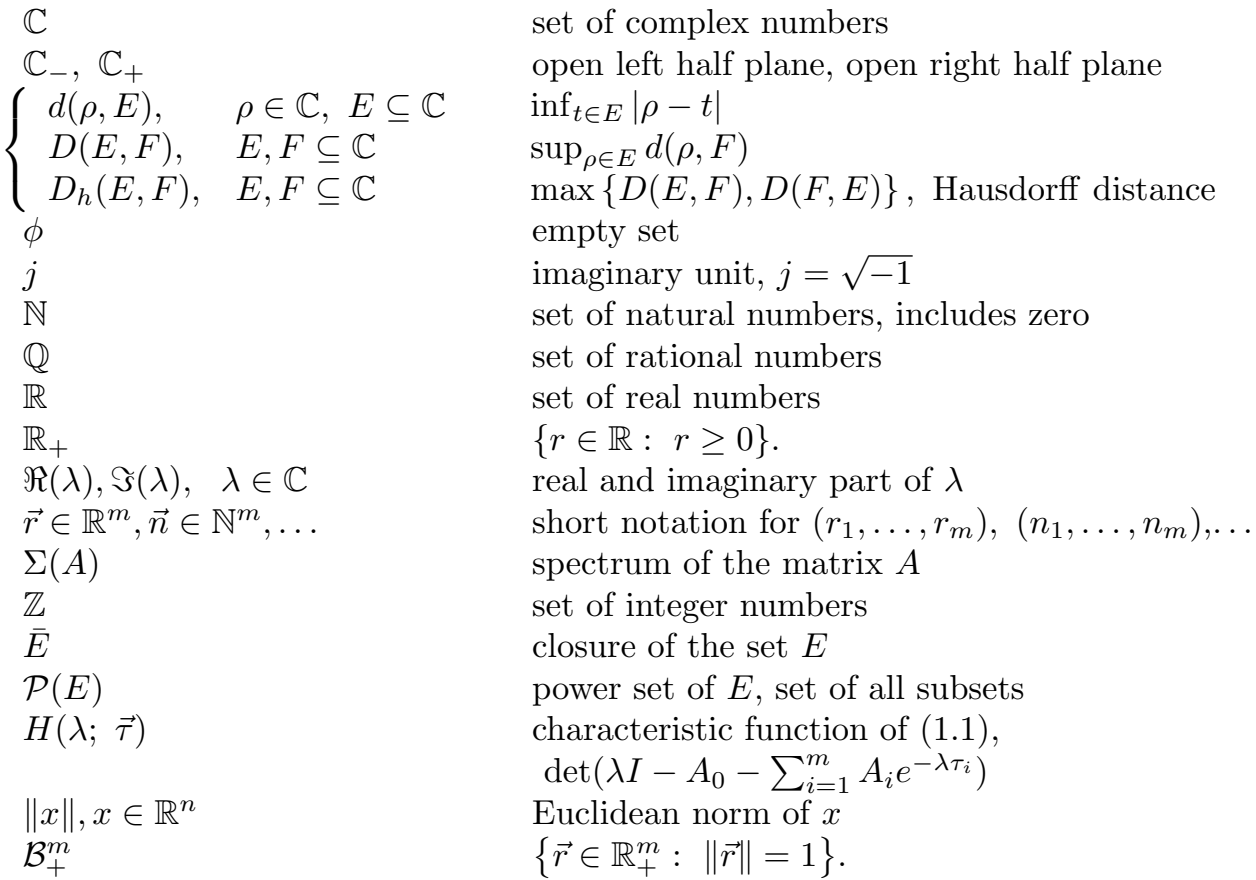

Given a direction $\left(r_{1}, \ldots, r_{m}\right) \in \mathcal{B}_{+}^{m}$ in the delay-parameter space, we define the associated ray, $\mathcal{T}(\vec{r})$, as follows:

Definition 2.1. For $\vec{r} \in \mathcal{B}_{+}^{m}$, let $\mathcal{T}(\vec{r}):=\left\{\tau_{0} \vec{r}: \tau_{0} \in \mathbb{R}_{+}\right\}$.

As we address delay-independent stability properties for both cases where all delays vary independently of each other, and where they are restricted to a particular ray, we will from now on consequently use the following terminology, in order to avoid confusion:

DeFinition 2.2. The system (1.1) is delay-independent stable if and only if its zero solution is asymptotically stable for all $\vec{\tau} \in \mathbb{R}_{+}^{m}$.

DeFinition 2.3. The ray $\mathcal{T}(\vec{r})$ is stable if and only if the zero solution of (1.1) is asymptotically stable for all $\vec{\tau} \in \mathcal{T}(\vec{r})$.

Regarding delay interference we have:

Definition 2.4. A stable ray $\mathcal{T}(\vec{r})$ is subjected to the delay interference phenomenon if and only if for all $\epsilon>0$, there exists a $\vec{s} \in \mathcal{B}_{+}^{m}$ with $\|\vec{r}-\vec{s}\|<\epsilon$ such that the ray $\mathcal{T}(\vec{s})$ is not stable.

Recall that the zero solution of (1.1) is asymptotically stable if and only if all its characteristic roots, the zeros of

$$
H(\lambda ; \vec{\tau})=\operatorname{det}\left(\lambda I-A_{0}-\sum_{i=1}^{m} A_{i} e^{-\lambda \tau_{i}}\right),
$$

are in the open left half plane. See, for instance, [14], for stability definitions and their relation with the location of the characteristic roots.

The following technical assumption will be made throughout the paper: 
Assumption 2.5 .

$$
\operatorname{det}\left(A_{0}+\sum_{i=1}^{m} A_{i}\right) \neq 0
$$

Note that if this assumption is not satisfied, we have

$$
\forall \vec{\tau} \in \mathbb{R}_{+}^{m} H(0 ; \vec{\tau})=0,
$$

which implies that the zero solution is not asymptotically stable, whatever the values of the delays. As the article is devoted to characterizations of delay-independent stability, the assumption can be made without loosing generality.

3. Preliminaries. The following sets and quantities will play a major role in the characterization of delay-independent stability and the interference phenomenon:

Definition 3.1. Let

$$
\begin{aligned}
& \mathbb{W}=\bigcup_{\vec{\theta} \in[0,2 \pi]^{m}} \Sigma\left(A_{0}+\sum_{i=1}^{m} A_{i} e^{-j \theta_{i}}\right), \\
& \alpha_{0}=\sup \{\Re(\lambda): \lambda \in \mathbb{W}\}
\end{aligned}
$$

and for $\vec{r} \in \mathcal{B}_{+}^{m}$, let

$$
\begin{aligned}
& \mathbb{V}(\vec{r})=\bigcup_{\theta \geq 0} \Sigma\left(A_{0}+\sum_{i=1}^{m} A_{i} e^{-j \theta r_{i}}\right), \\
& \alpha(\vec{r})=\sup \{\Re(\lambda): \lambda \in \mathbb{V}(\vec{r})\} .
\end{aligned}
$$

It directly follows that

$$
\begin{array}{ll}
\forall \vec{r} \in \mathbb{B}_{+}^{m} \quad & \overline{\mathbb{V}(\vec{r})} \subseteq \mathbb{W} \\
& \alpha(\vec{r}) \leq \alpha_{0} .
\end{array}
$$

As we shall see the relations (3.1) can be strict $(\subset$, resp. $<)$. However, if the components of $\vec{r}$ are rationally independent ${ }^{2}$, then the next proposition applies:

PROPOSITION 3.2. If the components of $\vec{r}$ are rationally independent, then

$$
\overline{\mathbb{V}(\vec{r})}=\mathbb{W}, \quad \alpha(\vec{r})=\alpha_{0} .
$$

Proof. Since $\mathbb{V}(\vec{r}) \subseteq \mathbb{W}$ and $\mathbb{W}$ is closed, it remains to prove for the first statement that every element of $W$ can be approximated arbitrarily well with elements from $\mathbb{V}(\vec{r})$.

For any $\tilde{\lambda} \in \mathbb{W}$ there exists a $\vec{\theta} \in[0,2 \pi]^{m}$ such that $\tilde{\lambda}$ is a zero of the polynomial

$$
p(\lambda):=\operatorname{det}\left(\lambda I-A_{0}-\sum_{i=1}^{m} A_{i} e^{-j \theta_{i}}\right) .
$$

Since the delays $\vec{r}$ are rationally independent, we can apply Kronecker's theorem [11, Theorem 444] which leads to: for all $n \geq 1$, there exists a $k_{n}>0$ such that

$$
\left|e^{-j \theta_{i}}-e^{-j k_{n} r_{i}}\right|<\frac{1}{n}, \quad i=1, \ldots, m
$$

\footnotetext{
${ }^{2}$ see Appendix B for definitions regarding the interdependency of numbers.
} 
It follows that the set of polynomials $\left\{p_{n}(\lambda)\right\}_{n \geq 1}$, where

$$
p_{n}(\lambda):=\operatorname{det}\left(\lambda I-A_{0}-\sum_{i=1}^{m} A_{i} e^{-j k_{n} r_{i}}\right)
$$

uniformly converges to $p(\lambda)$ on compact sets. Applying Theorem A1 of [18], a variant of Rouché's theorem, yields the following: for all $\epsilon>0$, there exists a $\bar{n} \geq 0$ such that for all $n \geq \bar{n}, p_{n}$ has a zero $\lambda_{n}$ satisfying $\left|\lambda_{n}-\tilde{\lambda}\right|<\epsilon$. Since for any $n$ the zeros of $p_{n}$ are contained in $\mathbb{V}(\vec{r})$ the proof the first statement is complete. The second statement is a direct corollary of the first.

Next, with $\mathcal{B}_{+}^{m}$ equipped with the Euclidean norm and $\mathcal{P}(\mathbb{C})$ with the Hausdorff metric, we address the continuity of the functions $\mathbb{V}(\cdot)$ and $\alpha(\cdot)$, see Appendix $\mathrm{A}$ for precise definitions. As we illustrate at the end of the section, these functions are in general not continuous at each point, however the following weaker property holds:

Proposition 3.3. The function $\mathbb{V}: \mathcal{B}_{+}^{m} \rightarrow \mathcal{P}(\mathbb{C}), \vec{r} \mapsto \mathbb{V}(\vec{r})$ is lower semicontinuous at each $\vec{r} \in \mathcal{B}_{+}^{m}$.

Proof. We prove lower semi-continuity of $\mathbb{V}(\cdot)$ at an arbitrary point $\vec{s} \in \mathcal{B}_{+}^{m}$.

Fix $\epsilon>0$. Put a grid on the complex plane, whose lines are parallel to the real or imaginary axis, and equally spaced with $\epsilon / 4$ in both directions. This partitions the complex plane into disjunct squares. If the closure of such a square has a nonempty intersection with $\mathbb{V}(\vec{s})$, we assign a complex number to it, obtained by sampling exactly one point from the intersection. Let the set $\mathcal{G}_{\epsilon}$ be defined as a collection of all these samples. Since $\mathbb{V}(\vec{r})$ is bounded we have $\operatorname{card}\left(\mathcal{G}_{\epsilon}\right)<\infty$, and by construction:

$$
\forall v \in \mathbb{V}(\vec{s}) \quad d\left(v, \mathcal{G}_{\epsilon}\right)<\epsilon / 2
$$

Next, take any point $g \in \mathcal{G}_{\epsilon}$. By definition, there exists a $\tilde{\theta}>0$ such that the matrix $A_{0}+\sum_{i=1}^{m} A_{i} e^{-j \tilde{\theta} s_{i}}$ has a characteristic root equal to $g$. From continuity properties of the characteristic roots, there exists a $\delta>0$ such that for all $\vec{r} \in \mathcal{B}_{+}^{m}$ with $\|\vec{r}-\vec{s}\|<\delta$, the matrix $A_{0}+\sum_{i=1}^{m} A_{i} e^{-j \tilde{\theta} r_{i}}$ has an eigenvalue, say $\lambda$, which satisfies $|\lambda-g|<\epsilon / 2$. This can be repeated for all point of $\mathcal{G}_{\epsilon}$, and, since the cardinality of this set is finite, we can choose the threshold $\delta$ independently of $g \in \mathcal{G}_{\epsilon}$. Hence, we obtain

$$
\exists \delta>0 \forall g \in \mathcal{G}_{\epsilon} \forall \vec{r} \in \mathcal{B}_{+}^{m}\|\vec{r}-\vec{s}\|<\delta \Rightarrow d(g, \mathbb{V}(\vec{r}))<\epsilon / 2
$$

The combination of (3.2) and (3.3) results in

$$
\exists \delta>0 \forall v \in \mathbb{V}(\vec{s}) \forall \vec{r} \in \mathcal{B}_{+}^{m} \quad\|\vec{r}-\vec{s}\|<\delta \Rightarrow d(v, \mathbb{V}(\vec{r}))<\epsilon
$$

Since the above analysis can be repeated for any $\epsilon>0$, we end up with

$$
\forall \epsilon>0 \exists \delta>0 \quad \forall \vec{r} \in \mathcal{B}_{+}^{m} \quad\|\vec{r}-\vec{s}\|<\delta \Rightarrow D(\mathbb{V}(\vec{s}), \mathbb{V}(\vec{r})) \leq \epsilon,
$$

that is, lower semi-continuity of $\mathbb{V}$ at $\vec{s}$. $\square$

Corollary 3.4. The function $\alpha: \mathcal{B}_{+}^{m} \rightarrow \mathbb{R}, \vec{r} \mapsto \alpha(\vec{r})$ is lower semi-continuous at each $\vec{r} \in \mathcal{B}_{+}^{m}$.

By combining Propositions 3.2 and 3.3 with relations (3.1) we arrive at a stronger result at rationally independent $\vec{r}$ : 
Proposition 3.5. The function $\mathbb{V}: \mathcal{B}_{+}^{m} \rightarrow \mathcal{P}(\mathbb{C}), \vec{r} \mapsto \mathbb{V}(\vec{r})$ is continuous at rationally independent $\vec{r}$. The function $\alpha: \mathcal{B}_{+}^{m} \rightarrow \mathbb{R}, \vec{r} \mapsto \alpha(\vec{r})$ is continuous at rationally independent $\vec{r}$.

We illustrate the above concepts and properties with the equation

$$
\dot{x}(t)=-1.3 x(t)-x\left(t-\tau_{1}\right)-\frac{1}{2} x\left(t-\tau_{2}\right),
$$

for which we have

$$
\begin{aligned}
& \mathbb{W}=\bigcup_{\left(\theta_{1}, \theta_{2}\right) \in[0,2 \pi]^{2}}\left(-1.3-e^{-j \theta_{1}}-\frac{1}{2} e^{-j \theta_{2}}\right)=\left\{\lambda \in \mathbb{C}: \quad \frac{1}{2} \leq|\lambda+1.3| \leq \frac{3}{2}\right\}, \\
& \mathbb{V}(\vec{r})=\bigcup_{\theta \geq 0}\left(-1.3-e^{-j r_{1} \theta}-\frac{1}{2} e^{-j r_{2} \theta}\right) .
\end{aligned}
$$

In Figure 3.1 we have shown the set $\mathbb{V}(\vec{r})$ for some values of $\vec{r}$. We have also indicated the boundaries of $\mathbb{W}$. When the components of $\vec{r}$ are commensurate, $\mathbb{V}(\vec{r})$ forms a closed curve. In all the cases displayed we have $\alpha(\vec{r})<\alpha_{0}$ and $\overrightarrow{\mathbb{V}(\vec{r})} \subset \mathbb{W}$ (strict relations). Discontinuities of the function $\vec{r} \mapsto \mathbb{V}(\vec{r})$ occur at all rationally dependent $\vec{r}$. To illustrate the mechanism, let us first compare the cases a.) and f.). Ratio $r_{2} / r_{1}=1.9$ can be seen as a perturbation of $r_{2} / r_{1}=2$ and, although the components of $\vec{r}$ are still commensurate, they are 'more independent', since the coprime numbers 19 and 10 (having ratio 1.9) are larger than 2 and 1 (having ratio 2). As a consequence, the curve

$$
\theta \geq 0 \mapsto-1.3-e^{-j \theta}-\frac{1}{2} e^{-j \frac{r_{2}}{r_{1}} \theta}
$$

with $r_{2} / r_{1}=1.9$ only closes at $\theta=20 \pi$ when $\theta$ is increased from zero (instead of $\theta=2 \pi$ for $r_{2} / r_{1}=2$ ), and a 'larger portion' of $\mathbb{W}$ is covered by $\mathbb{V}(\vec{r})$. This is clearly visible in subplot f.), where we have also depicted the values of (3.7) for $\theta \in[0,2 \pi]$ (bold part of curve). Next, if $r_{2} / r_{1}=2$ would be perturbed instead to an irrational value, such as $2-\pi / n, n \geq 2$, then the corresponding curve $\mathbb{V}(\vec{r})$ would never close and its points would densely fill $\mathbb{W}$, as follows from Proposition 3.2. Since $n$ can be taken arbitrarily large, the perturbation can be taken arbitrarily small.

4. Main results. Step-by-step we characterize the stability of the zero solution of (1.1) in the delay parameters, in terms of the sets $\mathbb{V}(\cdot)$ and $\mathbb{W}$. The main results will be stated in Theorem 4.1, Theorem 4.5 and Theorem 4.7, and they will be summarized in Table 4.2.

We first recall some properties of the characteristic roots of (1.1), which are needed in the subsequent analysis. The number of characteristic roots in any right half plane is finite. Furthermore, the function

$$
g: \mathbb{R}_{+}^{m} \rightarrow \mathbb{R}, \vec{\tau} \mapsto g(\vec{r})=\max _{\lambda \in \mathbb{C}}\{\Re(\lambda): H(\lambda ; \vec{\tau})=0\}
$$

is continuous [21]. As a consequence, if the zero solution of (1.1) is asymptotically stable for some delay values but the system is not delay-independent stable, then there exist delay values for which the rightmost characteristic roots are on the imaginary axis.

Necessary and sufficient conditions for delay-independent stability are expressed by the following theorem:

THEOREM 4.1. The system (1.1) is delay-independent stable if and only if

$$
\mathbb{W} \subset\left(\mathbb{C}_{-} \cup\{0\}\right)
$$


a.) $r_{2} / r_{1}=2$

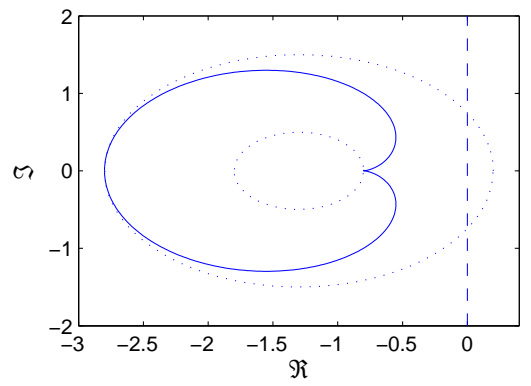

C.) $r_{2} / r_{1}=4$

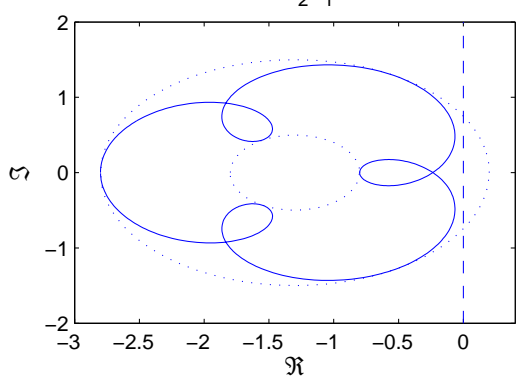

e.) $r_{2} / r_{1}=3 / 2$

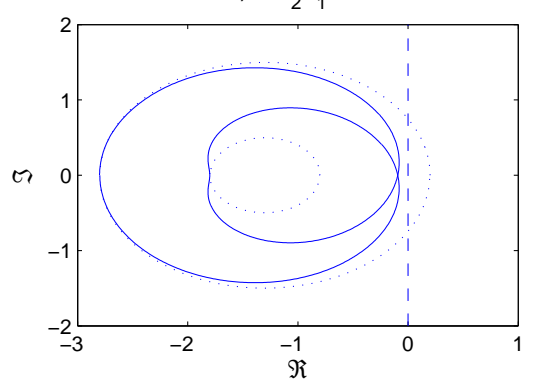

b.) $r_{2} / r_{1}=1 / 2$

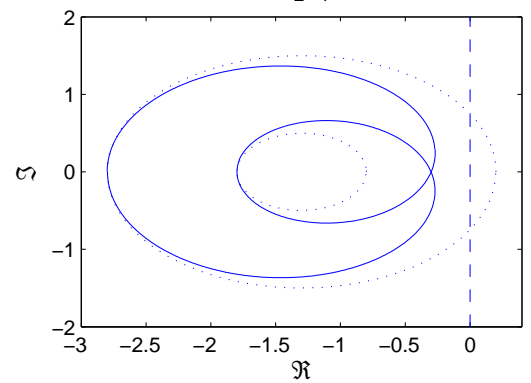

d.) $r_{2} / r_{1}=2 / 3$

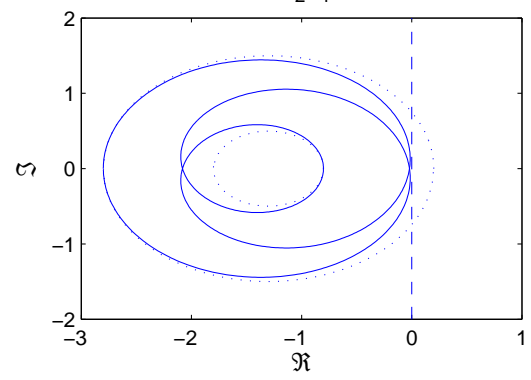

f.) $r_{2} / r_{1}=1.9$

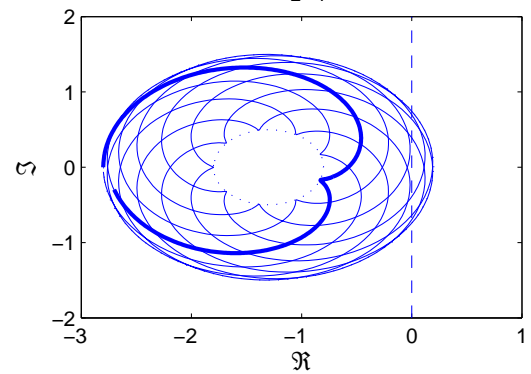

FIG. 3.1. The set $\mathbb{V}(\vec{r})$ for the system (3.6), for different values of $\vec{r}$ (solid curves). The dotted curves are the boundaries of $\mathbb{W}$.

Proof. Both implications are proven by contradiction.

" $\Rightarrow$ " Assume that $\mathbb{W} \not \subset\left(\mathbb{C}_{-} \cup\{0\}\right)$. We distinguish between two cases:

Case 1: $\mathbb{W}$ contains an element $j \omega, \omega \neq 0$, i.e. there exists a $\vec{\theta} \in\left[\begin{array}{ll}0,2 \pi]^{m} \\ \text { such that }\end{array}\right.$

$$
\operatorname{det}\left(j \omega I-A_{0}-\sum_{i=1}^{m} A_{i} e^{-j \theta_{i}}\right)=0 .
$$

It follows that $H\left(j \omega ; \vec{\tau}_{0}\right)=0$, where

$$
\vec{\tau}_{0}=\left(\frac{\theta_{1}}{\omega}, \ldots, \frac{\theta_{m}}{\omega}\right) .
$$


This contradicts the delay-independent stability.

Case $2: \mathbb{W}$ contains an element $\tilde{\lambda} \in \mathbb{C}_{+}$. Hence, there exists a $\vec{\theta}_{0} \in[0,2 \pi]^{m}$ such that

$$
A_{0}+\sum_{i=1}^{m} A_{i} e^{-j \theta_{i}}
$$

has a characteristic root $\tilde{\lambda}$ for $\vec{\theta}=\overrightarrow{\theta_{0}}$. If $\vec{\theta} \in \mathbb{R}_{+}^{m}$ is varied continuously (with respect to the Euclidean norm) from $\overrightarrow{\theta_{0}}$ to $\left(k_{1} 2 \pi, \ldots, k_{m} 2 \pi\right)$, for some $\vec{k} \in \mathbb{N}^{m}$, the spectrum of the matrix (4.3) continuously changes (w.r.t. the Hausdorff metric) to the spectrum of $A_{0}+\sum_{i=1}^{m} A_{i}$. If the latter matrix is not Hurwitz we have a contradiction (instability for all delays equal to zero). If not, it implies that $\mathbb{W} \cap \mathbb{C}_{+}$is not separated from the imaginary axis, and we are the situation described with Case 1.

" $\Leftarrow$ " Assume that (1.1) is not delay-independent stable. In the case where $A_{0}+$ $\sum_{i=1}^{m} A_{i}$ is not Hurwitz, this matrix has a characteristic root in the closed right half plane, different from zero (Assumption 2.5), and we already have a contradiction since $\Sigma\left(A_{0}+\sum_{i=1}^{m} A_{i}\right) \subseteq \mathbb{W}$. In the other case, the system (1.1) is asymptotically stable for all delays equal to zero and, by the continuity of (4.1), there must be characteristic roots on the imaginary axis for some delay values, i.e.

$$
\exists \omega>0 \exists \vec{\tau}_{0} \in \mathbb{R}_{+}^{m} H\left(j \omega ; \vec{\tau}_{0}\right)=0 .
$$

It follows that $j \omega \in \mathbb{W}$ and we have a contradiction.

REMARK 4.2. For a delay-independent stable system, the condition $0 \notin \mathbb{W}$, respectively $0 \in \mathbb{W}$, is referred to in the literature as strong, respectively weak delayindependent stability (see [2, 21] and the references therein), notions which are related to stability properties of some associated $2 D$-system.

The stability of a ray $\mathcal{T}(\vec{r})$ can be characterized in an analogous way:

Proposition 4.3. The ray $\mathcal{T}(\vec{r})$ is stable if and only if

$$
\mathbb{V}(\vec{r}) \subset\left(\mathbb{C}_{-} \cup\{0\}\right) .
$$

Proof. Similar to the proof of Theorem 4.1.

The next proposition states that if the system (1.1) is not delay-independent stable, then it is always prone to the delay interference phenomenon:

Proposition 4.4. Assume that $\mathbb{W} \not \subset\left(\mathbb{C}_{-} \cup\{0\}\right)$. If a ray $\mathcal{T}(\vec{r})$ is stable, then it is subjected to the delay interference phenomenon.

Proof. From the assumption $\mathbb{W} \not \subset\left(\mathbb{C}_{-} \cup\{0\}\right)$ it follows that $(1.1)$ is not delayindependent stable. So there exist delay values $\vec{\tau} \in \mathbb{R}_{+}^{m}$ and a frequency $\omega>0$ such that $H(j \omega ; \vec{\tau})=0$. For any given $\vec{k} \in \mathbb{N}^{m}, \vec{k} \neq 0$, this implies that $H(j \omega ; \vec{\tau}(n))=0$ for all $n \in \mathbb{N}$, where

$$
\vec{\tau}(n)=\left(\tau_{1}+k_{1} n \frac{2 \pi}{\omega}, \ldots, \tau_{m}+k_{m} n \frac{2 \pi}{\omega}\right) .
$$

Therefore, the ray

$$
\mathcal{T}\left(\frac{\vec{\tau}(n)}{\|\vec{\tau}(n)\|}\right)
$$


is unstable for all $n \in \mathbb{N}$. From

$$
\lim _{n \rightarrow \infty} \frac{\vec{\tau}(n)}{\|\vec{\tau}(n)\|}=\frac{\vec{k}}{\|\vec{k}\|}
$$

and the fact that this analysis can be repeated for every nonzero $\vec{k} \in \mathbb{N}^{m}$, it follows that every ray $\mathcal{T}(\vec{r})$ where $\vec{r}$ has commensurate components, gets unstable when applying certain infinitesimal perturbations to $\vec{r}$.

If the components of $\vec{r}$ are non-commensurate, then there exist infinitesimal $\delta \vec{r}$ such that $\vec{r}+\delta \vec{r}$ has commensurate components $\left(\mathbb{Q}^{m}\right.$ is dense in $\left.\mathbb{R}^{m}\right)$, and the above arguments can be repeated. This completes the proof. $\square$

Combining the previous results for the case where $\mathbb{W}$ has a non-empty intersection with the open right half plane yields:

TheOrem 4.5. Assume that $\mathbb{W} \cap \mathbb{C}_{+} \neq \phi$. Then the following holds:

1. If the components of $\vec{r}$ are rationally independent, then the ray $\mathcal{T}(\vec{r})$ is unstable.

2. if the ray $\mathcal{T}(\vec{r})$ is stable, then it is subjected to the delay interference phenomenon.

3. The set $\left\{\vec{r} \in \mathcal{B}_{+}^{m}: \mathcal{T}(\vec{r})\right.$ stable $\}$ is nowhere dense in $\mathcal{B}_{+}^{m}$.

Proof. If the components of $\vec{r}$ are rationally independent, then $\overline{\mathbb{V}(\vec{r})}=\mathbb{W}$ by Proposition 3.2. A combination with the assumption of the theorem leads to $\mathbb{V}(\vec{r}) \cap$ $\mathbb{C}_{+} \neq \phi$. Applying Proposition 4.3 then yields the first assertion.

The second assertion can be proven in two different ways. It directly follows from Proposition 4.4, whose proof mainly relies on the invariance property (4.5). Alternatively, let $\vec{r} \in \mathbb{R}_{+}^{m}$ be such that $\mathcal{T}(\vec{r})$ is stable. Following from the density of the rationally independent numbers in $\mathbb{C}^{m}$, there exist arbitrarily small perturbations $\delta \vec{r} \in \mathbb{R}_{+}^{m}$ such that $\vec{r}+\delta \vec{r}$ has rationally independent components. By the first assertion of the theorem, the ray $\mathcal{T}((\vec{r}+\delta \vec{r}) /(\|\vec{r}+\delta \vec{r}\|))$ is unstable. This implies that $\mathcal{T}(\vec{r})$ is subjected to the delay interference phenomenon.

The third assertion follows from Propositions 3.2-3.3 and Proposition 4.3.

Under the assumption of the previous theorem, we comment on the detection of stable rays $\mathcal{T}(\vec{r})$ (if any), without the explicit computation of stability/instability regions of (1.1) in the delay-parameter space. From Theorem 4.5, the components of $\vec{r}$ can not be rationally independent. By Proposition 4.3, we have to search for values for which $\mathbb{V}(\vec{r}) \subset\left(\mathbb{C}_{-} \cup\{0\}\right)$, whereas we have $\mathbb{W} \cap \mathbb{C}_{+} \neq \phi$ and $\mathbb{V}(\vec{r}) \subseteq \mathbb{W}$. Thus, values of $\vec{r}$, for which $\mathbb{V}(\vec{r})$ "doesn't cover $\mathbb{W}$ very well", are good candidates. Following from Propositions 3.2 and 3.3, such values must be characterized by a "large dependence", for instance, $\vec{r}=\vec{n} /\|\vec{n}\|$, with $\vec{n} \in \mathbb{N}^{m}$ and $\|\vec{n}\|$ small. To fix the ideas, we pick up the example (3.6), which satisfies $\mathbb{W} \cap \mathbb{C}_{+} \neq \phi$ since $\alpha_{0}=0.2$. Table 4.1 displays the corresponding values of $\alpha(\vec{r})$, for rationally dependent $\vec{r}$. It becomes apparent that stable rays correspond to

$$
\left(n_{1}, n_{2}\right) \in\{(1,0),(0,1),(1,2),(1,4),(2,1),(2,3),(3,2)\} .
$$

Notice that for five of these cases the set $\mathbb{V}(\vec{r})$ is depicted in Figure 3.1, subplots a.) to e.).

Finally, we look at the special case, where $\mathbb{W} \not \subset\left(\mathbb{C}_{-} \cup\{0\}\right)$, but still lies in the closed left half plane. But first we prove the following lemma: 


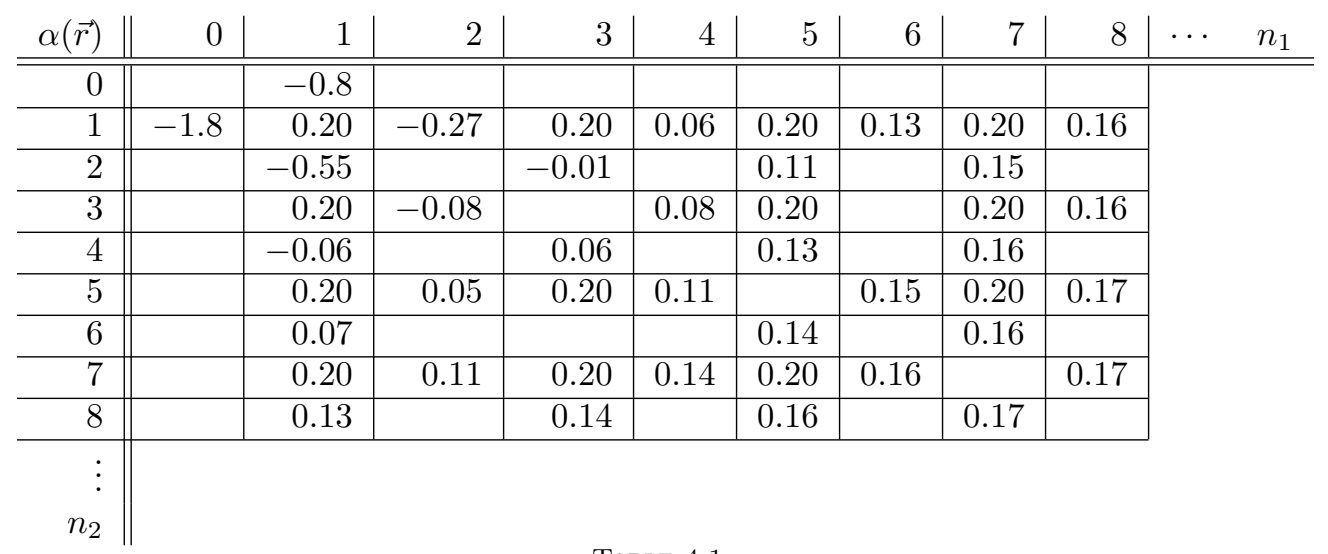

TABLE 4.1

Values of $\alpha(\vec{r})$, where $\vec{r}=\left(n_{1}, n_{2}\right) / \sqrt{n_{1}^{2}+n_{2}^{2}}$, computed for the system (3.6).

Lemma 4.6. Assume that (1.1) has a characteristic root in $\mathbb{C}_{+}$for some delay values. Then $\mathbb{W} \cap \mathbb{C}_{+} \neq \phi$.

Proof. Inspired by the proof of Lemma 2.2 in [10], we use an approximation and a continuation argument.

Let $\vec{\tau}$ be delay values, for which (1.1) has a characteristic root $\tilde{\lambda} \in \mathbb{C}_{+}$, i.e.

$$
\operatorname{det}\left\{\tilde{\lambda} I-A_{0}-\sum_{i=1}^{m} A_{i} e^{-\tilde{\lambda} \tau_{i}}\right\}=0 .
$$

Without loosing generality we may assume that the components of $\vec{\tau}$ are commensurate $^{3}$, and we can set

$$
\vec{\tau}=\tau_{0}\left(\frac{p_{1}}{q}, \ldots, \frac{p_{m}}{q}\right), \quad p_{i} \in \mathbb{N}, i=1, \ldots, m, \quad q \in \mathbb{N} .
$$

Then (4.7) can be written as

$$
\operatorname{det}\left\{\tilde{\lambda} I-A_{0}-\sum_{i=1}^{m} A_{i} \mu_{0}^{p_{i}}\right\}=0
$$

where

$$
\mu_{0}=e^{-\frac{\tilde{\lambda} \tau_{0}}{q}}
$$

From (4.8) it follows that the polynomial

$$
p(\mu ; k):=\operatorname{det}\left\{k \tilde{\lambda} I-A_{0}-\sum_{i=1}^{m} A_{i} \mu^{p_{i}}\right\}
$$

parameterized by $k \in[1, \infty)$, satisfies $p\left(\mu_{0} ; 1\right)=0$. By definition we have $\left|\mu_{0}\right|<1$. Obviously, as $k \rightarrow \infty$, all zeros of (4.9) must become unbounded. From this and the

\footnotetext{
${ }^{3}$ If not, they can be made commensurate by adding perturbations, which can be chosen sufficiently small, such that characteristic roots in $\mathbb{C}_{+}$are maintained.
} 


$\left.\begin{array}{|c|c|c|}\hline \mathbb{W} \subset\left(\mathbb{C}_{-} \cup\{0\}\right) & \mathbb{W} \not \subset\left(\mathbb{C}_{-} \cup\{0\}\right) \text { and } \\ \mathbb{W} \cap \mathbb{C}_{+}=\phi\end{array}\right)$

Characterization of stability of the steady state solution of (1.1) along rays in the delay parameter space, as a function of $\mathbb{W}$ and $\mathbb{V}(\cdot)$.

continuity of the zeros w.r.t. $k$, there must be a pair $(\tilde{k}, \tilde{\mu})$ with $\tilde{k}>1$ and $|\tilde{\mu}|=1$ such that

$$
\operatorname{det}\left\{\tilde{k} \tilde{\lambda} I-A_{0}-\sum_{i=1}^{m} A_{i} \tilde{\mu}^{p_{i}}\right\}=0 .
$$

This means that $\tilde{k} \tilde{\lambda} \in \mathbb{W}$ and, consequently, $\mathbb{W} \cap \mathbb{C}_{+} \neq \phi$.

The combination of Theorem 4.1, Proposition 4.4 and Lemma 4.6 results in:

Theorem 4.7. Assume that $\mathbb{W} \cap \mathbb{C}_{+}=\phi$ and $\mathbb{W} \not \subset\left(\mathbb{C}_{-} \cup\{0\}\right)$. Then there exist delay values for which (1.1) has characteristic roots on the imaginary axis, while there are no delay values for which (1.1) has characteristic roots in $\mathbb{C}_{+}$. Every stable ray is subjected to the delay interference phenomenon.

The main results of this section are summarized in Table 4.2. The cases $\mathbb{W} \cap \mathbb{C}_{+} \neq$ $\phi$ and $\mathbb{W} \subset\left(\mathbb{C}_{-} \cup\{0\}\right)$ with $0 \notin \mathbb{W}$ are generic. The other cases, where the rightmost elements of $\mathbb{W}$ are on the imaginary axis, characterize situations where a system is on the edge of loosing or acquiring delay-independent stability. Recall that this analysis has been performed under Assumption 2.5, which can be checked a priori (if the assumption is not satisfied, then there exist no values of $\vec{\tau}$ for which the zero solution of (1.1) is asymptotically stable).

To conclude, we briefly discuss some existing results related to the interference phenomenon. In [15] it is shown that delay-independent stability is equivalent to asymptotic stability for all delay values lying in a nontrivial sector in the delayparameter space, and to the robustness of stability of a ray, consisting of commensurate delay values, w.r.t. small perturbations of the direction (see [15] for precise formulations). Note that the latter two statements imply the existence of a stable ray, which is not subjected to the interference phenomenon. Given a stable ray, the additional condition to have interference of [17] (delay-dependent stability when one of the (two) delays is set to zero) and of [22] (frequency-sweeping test) are in fact conditions for the presence of characteristic roots on the imaginary axis for some delay values, thus conditions for not having delay-independent stability. In this way, the above cited results are a direct corollary of the fact that a system is either delay-independent stable or every stable ray is subjected to the delay interference phenomenon, see Table 4.2.

5. Illustrations. We present two examples, which together illustrate all results and phenomena described in the previous sections. Besides the delays, the examples will exhibit another parameter. This allows us to illustrate, in addition, two scenarios 
for the transition between delay-independent stability and delay-dependent stability: one via weak delay-independent stability, the other via the case $\mathbb{W} \not \subset\left(\mathbb{C}_{-} \cup\{0\}\right), \mathbb{W} \cap$ $\mathbb{C}_{+}=\phi$.

As a first example we consider

$$
\dot{x}(t)=-a x(t)-x\left(t-\tau_{1}\right)-\frac{1}{2} x\left(t-\tau_{2}\right)
$$

where $a \in \mathbb{R}$ is a parameter. We have

$$
\mathbb{W}=\left\{\lambda \in \mathbb{C}: \frac{1}{2} \leq|\lambda+a| \leq \frac{3}{2}\right\}
$$

According to Table 4.2 the zero solution of (5.1) is delay-independent stable if and only if $a \geq \frac{3}{2}$. For $a>\frac{3}{2}$ we have strong delay-independent stability, for $a=\frac{3}{2}$ we have weak delay-independent stability. For $a<\frac{3}{2}$ we are directly in the case $\mathbb{W} \cap \mathbb{C}_{+} \neq \phi$.

For $a=1.3$ the system reduces to (3.6), for which the analysis in the previous section and Table 4.1 learn that there are 7 stable rays, characterized by $\vec{r}=\vec{n} /\|\vec{n}\|$, with $\vec{n}$ given by (4.6). Such an analysis can easily be repeated for other values of $a$, since a change of this parameter, say $\delta a$, only affects the sets $\mathbb{W}$ and $\mathbb{V}(\vec{r})$ by a shift along the real axis of $-\delta a$, while the numerical values of Table 4.1 change accordingly with $-\delta a$. For $a=1$, there are only three stable rays left, characterized by

$$
\left(n_{1}, n_{2}\right) \in\{(1,0),(0,1),(1,2)\}
$$

and for $a<-0.5$ all rays are unstable.

In Figure 5.1 we show the complete stability/instability regions of (5.1) in the delay-parameter space ${ }^{4}$, for $a=1$ and $a=1.3$. The solid lines correspond to delay values for which there are characteristic roots on the imaginary axis. The dashed lines indicate the stable rays. Notice that small perturbations of their slope lead to intersections with the solid curves, a consequence of the delay interference phenomenon. As $a \rightarrow 1.5$, we have in fact a scenario towards delay-independent stability, characterized by an increase of the number of stable rays. That number becomes arbitrarily large when getting arbitrarily closed to the 'bifurcation value' $a=1.5$, for which we have weak delay-independent stability.

Next, we analyze the system

$$
\dot{x}(t)=A_{0} x(t)+A_{1} x\left(t-\tau_{1}\right)+A_{2} x\left(t-\tau_{2}\right),
$$

where

$$
A_{0}=\left[\begin{array}{cc}
0 & 1 \\
a-a^{2}-\frac{5}{4} & 2 a-1
\end{array}\right], \quad A_{1}=\left[\begin{array}{cc}
0 & 0 \\
\frac{1}{5} & 0
\end{array}\right], \quad A_{2}=\left[\begin{array}{cc}
0 & 0 \\
-\frac{4}{5} & 0
\end{array}\right]
$$

and $a \in \mathbb{R}$ is a parameter. The corresponding set $\mathbb{W}$ is depicted in Figure 5.2. A change of parameter $a$ results once again in a shift of this set along the real axis.

\footnotetext{
${ }^{4}$ The stability crossing curves were computed as Hopf bifurcation curves with the package DDEBIFTOOL [7], thereby exploiting (frequency dependent) invariance properties w.r.t. delay shifts. Other approaches are described in [9] and [23].
} 

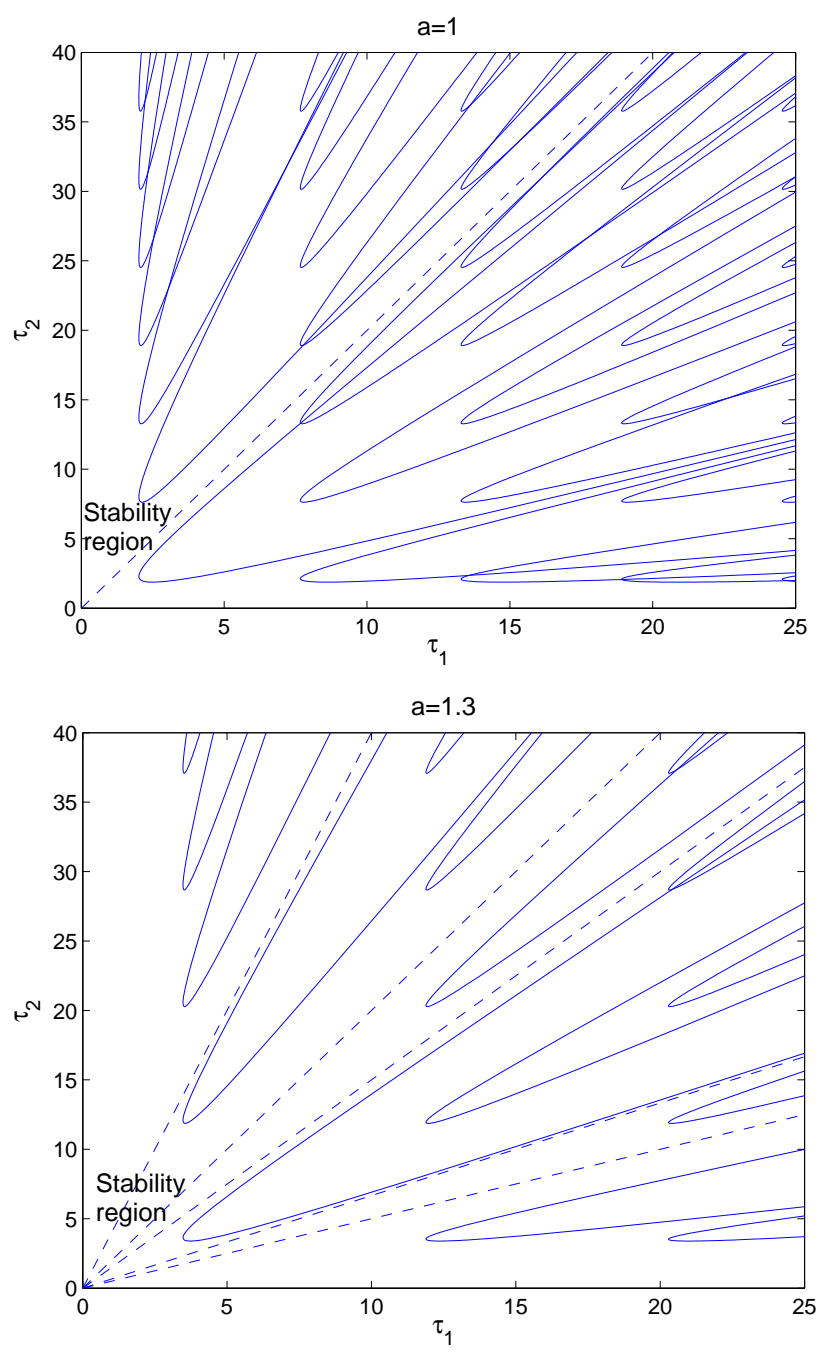

FIG. 5.1. Stability/instability regions of the zero solution of (5.1) in the $\left(\tau_{1}, \tau_{2}\right)$-space, for a $=1$ (top) and $a=1.3$ (bottom). For $a=1$, the directions of the stable rays (dashed lines) are given by (5.2); for $a=1.3$ by (4.6). For $a \geq 1.5$ the systems is delay-independent stable.

For $a<0$ the system is strongly delay-independent stable and for $a>0$ we have $\mathbb{W} \cap \mathbb{C}_{+} \neq \phi$, see Table 4.2. For the intermediate value, $a=0$, we are in the special case where the assumptions of Theorem 4.7 are satisfied.

In Figure 5.3, we plot the stability region in the $\left(\tau_{1}, \tau_{2}\right)$-space for $a=1 / 16$ and $a=0$. In the first case there are three stable rays, determined by $\vec{r}=\vec{n} /\|\vec{n}\|$, with

$$
\vec{n} \in\{(1,0),(1,1),(4,1)\} .
$$

These directions can also be obtained directly by constructing a table similar to Table 4.1. As $a \rightarrow 0+$, all closed curves in the $\left(\tau_{1}, \tau_{2}\right)$-space, corresponding to characteristic roots on the imaginary axis, shrink, and at the limit $a=0$, where Theorem 4.7 applies, they have collapsed to equally spaced points. For such delay values, which 


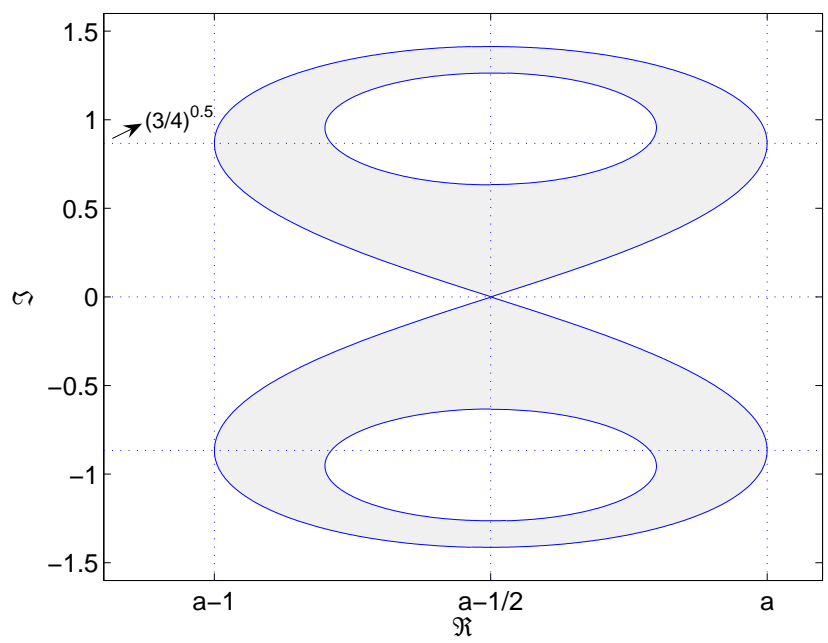

FIG. 5.2. The set $\mathbb{W}$, corresponding to the system (5.3)-(5.4), is shaded.

can be computed analytically as

$$
\left(\tau_{1}, \tau_{2}\right)=\left(\frac{10 \pi}{3 \sqrt{3}}+k \frac{4 \pi}{\sqrt{3}}, \frac{4 \pi}{3 \sqrt{3}}+l \frac{4 \pi}{\sqrt{3}}\right), \quad k, l \in \mathbb{N},
$$

the system has characteristic roots on the imaginary axis (more precisely $\pm j \sqrt{3} / 2$ ), but for all other delay values its zero solution is asymptotically stable. This is illustrated in Figure 5.4, where the real parts of the rightmost characteristic roots are displayed along a particular ray, containing some of the points (5.5).

Notice that for the limit case, $a=0$, the number of unstable rays is infinite but countable, since the unstable rays have to contain at least one of the points (5.5). Hence, they are characterized by a slope

$$
\frac{r_{2}}{r_{1}}=\frac{2+6 l}{5+6 k}, k, l \in \mathbb{N},
$$

being rational numbers. This leads to a paradox: for $a=0$ all rays $\mathcal{T}(\vec{r})$, with $r_{2} / r_{1}$ irrational, are stable, whereas for any $a>0$, they are unstable (since $\mathbb{W} \cap \mathbb{C}_{+} \neq \phi$ and Theorem 4.5 applies). The explanation is as follows: for all rationally independent $\vec{r}$ and every value of $a$, we have for the example:

$$
\begin{aligned}
& \alpha(\vec{r})=\alpha_{0}, \\
& \Re(\lambda)<\alpha_{0}, \forall \lambda \in \mathbb{V}(\vec{r}) .
\end{aligned}
$$

Consequently, $\mathbb{V}(\vec{r})$ intersects the closed right half plane if $\alpha_{0}>0$ but not if $\alpha_{0}=0$.

6. Relation with the stability of continuous-time delay-difference equations. We relate the results of the previous sections with stability conditions for continuous-time delay-difference equations of the form:

$$
x(t)-\sum_{i=1}^{m} A_{i} x\left(t-r_{i}\right)=0,
$$



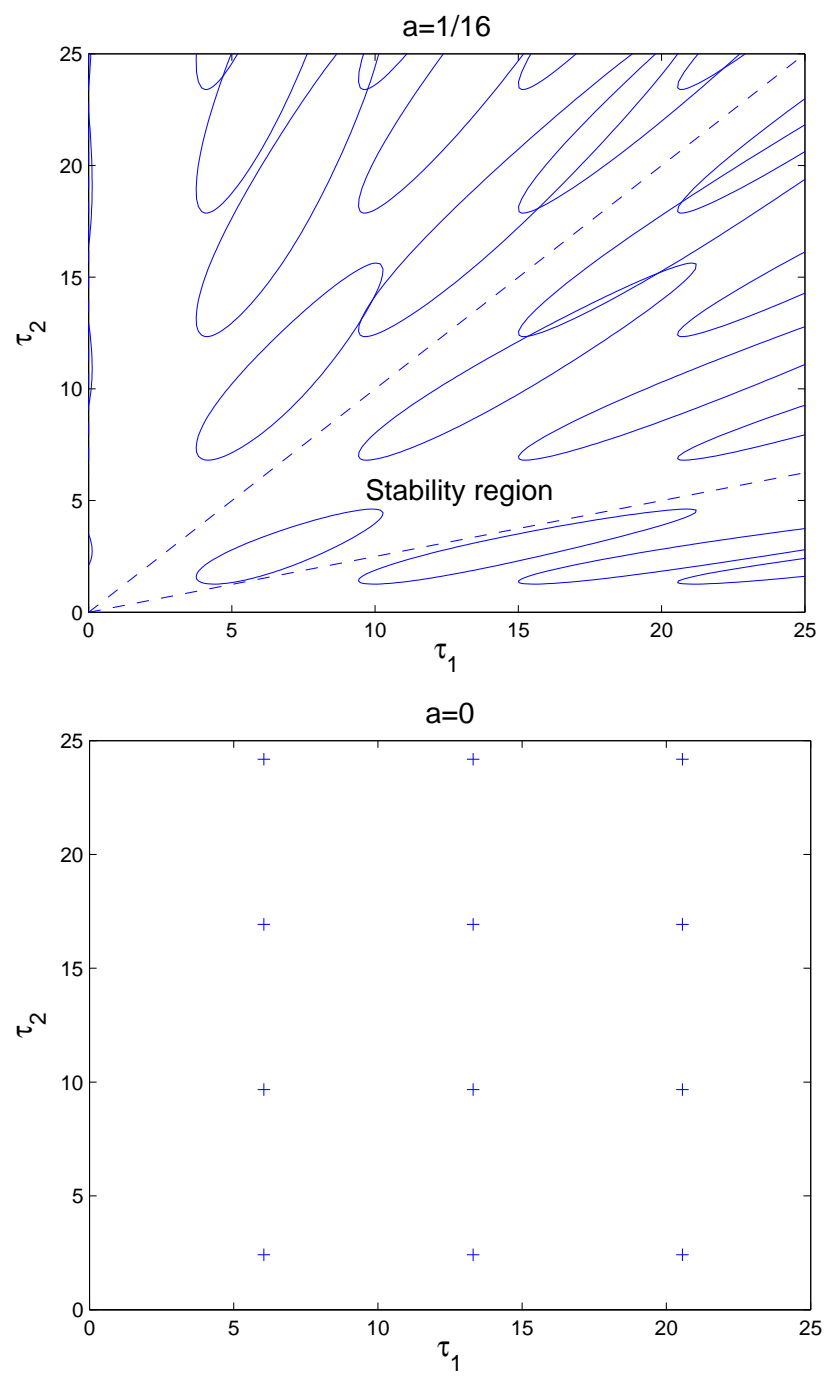

FIG. 5.3. Stability/instability regions of the zero solution of (5.3)-(5.4) in the $\left(\tau_{1}, \tau_{2}\right)$-space, for $a=1 / 16$ (top) and $a=0$ (bottom).

which are discussed in e.g. $[10,19,18]$ and the references therein.

We assume that $\operatorname{det}\left(I-\sum_{i=1}^{m} A_{i}\right) \neq 0$, which prevents a characteristic root at zero for all $\vec{r} \in \mathbb{R}_{+}^{m}$. Then the stability conditions for the zero solution of (6.1) from [10, Theorem 2.2 and Corollary 2.2] can be rephrased as displayed in Table 6.1.

A comparison between Table 4.2 and Table 6.1 reveals a strong correspondence between the stability of the zero solution of (1.1) along rays in the delay space, and the stability of the zero solution of (6.1). This correspondence is described in Table 6.2.

7. Concluding remarks. A complete characterization of delay-independent stability, stability of rays in the delay-parameter space, and the delay interference phenomenon was made. The results were illustrated with numerical examples. In addition, scenarios for the transition between delay-dependent stability and delay- 


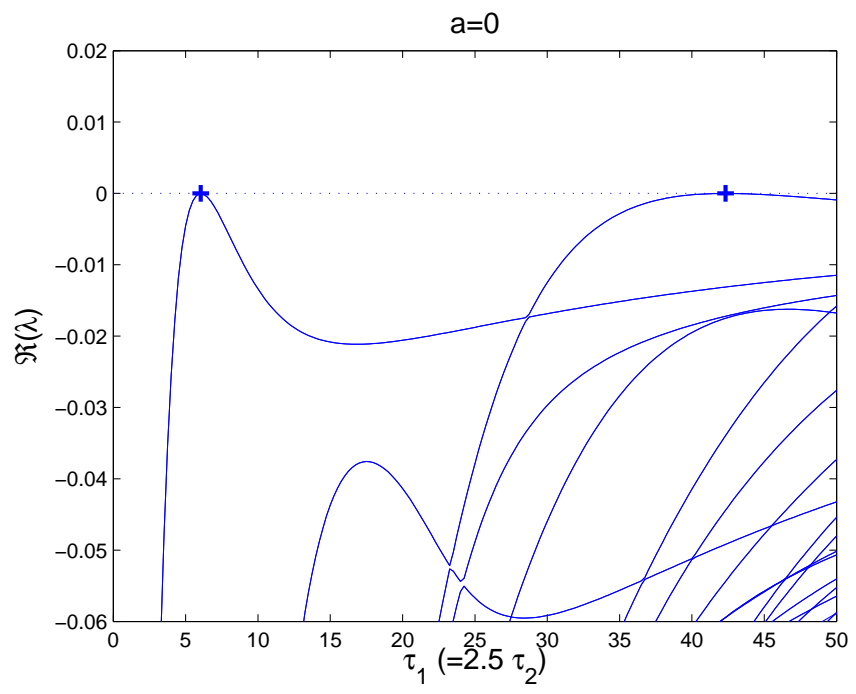

FIG. 5.4. Real parts of the rightmost characteristic roots of the system (5.3)-(5.4) with $a=0$, for delay values along the ray $\mathcal{T}(5 / \sqrt{29}, 2 / \sqrt{29})$.

\begin{tabular}{|l|l|}
\hline $\mathbb{W} \subset\{\lambda \in \mathbb{C}:|\lambda|<1\}$ & $\sup \{|\lambda|: \lambda \in \mathbb{W}\}>1$ \\
\hline \hline asympt. stable for all values of $\vec{r}$ & not asympt. stable for all values of $\vec{r}$ \\
\hline & if $\vec{r}$ is stabilizing, then there exist \\
& infinitesimal perturbations on $\vec{r}$ which \\
& destroy stability $\equiv$ not strongly stable \\
\cline { 2 - 2 } & unstable for rationally independent $\vec{r}$ \\
\hline
\end{tabular}

Conditions for the stability of the zero solution of (6.1). The set $\mathbb{W}$ is defined as in Definition 3.1, with $A_{0}$ set to zero.

independent stability were shown, and the correspondence with the stability theory for delay-difference equations was outlined.

It is worthwhile to mention that the stability results for rays in the delay-parameter space can easily be extended towards higher dimensional subspaces. For instance, asymptotic stability for all delay values lying in a subspace spanned by $\vec{r}^{(1)} \in \mathcal{B}_{+}^{m}$ and $\vec{r}^{(2)} \in \mathcal{B}_{+}^{m}$ will be determined by the position of the set

$$
\bigcup_{\theta_{1}, \theta_{2} \geq 0} \Sigma\left(A_{0}+\sum_{i=1}^{m} A_{i} e^{-j\left(\theta_{1} r_{i}^{(1)}+\theta_{2} r_{i}^{(2)}\right)}\right) \subseteq \mathbb{W}
$$

Furthermore, the study of the sets $\mathbb{W}$ and $\mathbb{V}(\cdot)$ does not only lead to a powerful tool for characterizing delay-independent stability properties. Note for instance that the intersection of $\mathbb{W}$ with the imaginary axis contains all possible values where characteristic roots can cross the imaginary axis when the delays are varied. Actually, from these values and/or the corresponding parameters, that is, $\vec{\theta}$ in Definition 3.1, detailed information can be retrieved about the geometry of the stability crossing curves/(hyper)surfaces in the delay-parameter space, thereby generalizing the results from $[9]$. 


\begin{tabular}{|lll|}
\hline Equation $(1.1)$ & $\rightsquigarrow$ & Equation $(6.1)$ \\
\hline relation of $\mathbb{W}$ w.r.t. imaginary axis & $\rightsquigarrow$ & relation of $\mathbb{W}$ w.r.t. unit circle \\
direction $\vec{r}$ in delay space & $\rightsquigarrow$ & nominal delay value $\vec{r}$ \\
(un)stable ray $\mathcal{T}(\vec{r})$ & $\rightsquigarrow$ & (de)stabilizing delay value $\vec{r}$ \\
stable ray subjected to the delay & $\rightsquigarrow$ & stability for nominal $\vec{r}$ destroyed by \\
interference phenomenon & & infinitesimal perturbations on $\vec{r}$ \\
\hline \hline
\end{tabular}

The similarity between the stability of the zero solution of (1.1) along rays in the delay space, and the stability of the zero solution of (6.1).

Acknowledgements. The authors would like to thank the Associate Editor and the anonymous reviewers for the careful reading of the manuscript and the careful comments.

This article presents results of the research project IAP P5, funded by the programme on Interuniversity Poles of Attraction, initiated by the Belgian State, Prime Minister's Office for Science, Technology and Culture, and of the bilateral research project PAI Tournesol (France, Flemish Community) "Distributed delays in dynamical systems: Analysis and applications" (2006-2007). Wim Michiels is a postdoctoral fellow of the Fund for Scientific Research -Flanders (Belgium).

Appendix A. Continuity properties. Throughout the paper we encounter functions from $\mathcal{B}_{+}^{m} \subset \mathbb{R}^{m}$ to $\mathbb{R}$ and from $\mathcal{B}_{+}^{m}$ to $\mathcal{P}(\mathbb{C})$. We assume that $\mathbb{R}^{m}$ is equipped with the Euclidean norm and $\mathcal{P}(\mathbb{C})$ with the Hausdorff metric.

A function $f: \mathcal{B}_{+}^{m} \rightarrow \mathbb{R}$ is lower semi-continuous, respectively upper semi-continuous at $\vec{s}$ if and only if (see, for instance, [1])

$$
\forall \epsilon>0 \exists \delta>0 \quad \forall \vec{r} \in \mathcal{B}_{+}^{m}\|\vec{r}-\vec{s}\|<\delta \Rightarrow f(\vec{r})-f(\vec{s})>-\epsilon,
$$

respectively

$$
\forall \epsilon>0 \exists \delta>0 \quad \forall \vec{r} \in \mathcal{B}_{+}^{m} \quad\|\vec{r}-\vec{s}\|<\delta \Rightarrow f(\vec{r})-f(\vec{s})<\epsilon .
$$

It is continuous at $\vec{s}$ when it is both upper and lower semi-continuous at $\vec{s}$.

A function $f: \mathcal{B}_{+}^{m} \rightarrow \mathcal{P}(\mathbb{C})$ is lower semi-continuous at $\vec{s}$ if and only if

$$
\forall \epsilon>0 \exists \delta>0 \quad \forall \vec{r} \in \mathcal{B}_{+}^{m}\|\vec{r}-\vec{s}\|<\delta \Rightarrow D(f(\vec{s}), f(\vec{r}))<\epsilon .
$$

When replacing $D(f(\vec{s}), f(\vec{r}))$ with $D(f(\vec{r}), f(\vec{s}))$, respectively $D_{h}(f(\vec{s}), f(\vec{r}))$, we have upper semi-continuity, respectively continuity at $\vec{s}$.

Appendix B. Inderdependency of numbers. The real numbers $r_{1}, r_{2}, \ldots, r_{m}$ are rationally independent if and only if

$$
\sum_{i=1}^{m} z_{i} r_{i}=0, \quad z_{i} \in \mathbb{Z},
$$

implies $z_{i}=0, \quad i=1, \ldots, m$. For example, two numbers are rationally independent if and only if their ratio is an irrational number. 
If the real numbers $r_{1}, \ldots r_{m}$ are rationally dependent (that is, not rationally independent), then there always exists an integer $p<m$ and a matrix $\Gamma \in \mathbb{Z}^{m \times p}$ of full column rank such that

$$
\left[\begin{array}{c}
r_{1} \\
\vdots \\
r_{m}
\end{array}\right]=\Gamma\left[\begin{array}{c}
s_{1} \\
\vdots \\
s_{p}
\end{array}\right]
$$

with the numbers $s_{1}, \ldots, s_{p}$ rationally independent. Thus, rationally dependent numbers depend on a smaller number of rationally independent numbers. In the special case where $p=1$, the numbers $r_{1}, \ldots, r_{m}$ are called commensurate, as they are all multiples of the same number.

For example, the numbers $1, \pi$ and $\exp (1)$ are rationally independent, the numbers 1,2 and $5 / 3$ commensurate. The numbers $1, \pi$ and $1+\pi$ are rationally dependent, yet not commensurate, as

$$
\left[\begin{array}{c}
1 \\
\pi \\
1+\pi
\end{array}\right]=\left[\begin{array}{ll}
1 & 0 \\
0 & 1 \\
1 & 1
\end{array}\right]\left[\begin{array}{l}
1 \\
\pi
\end{array}\right]
$$

with 1 and $\pi$ rationally independent.

\section{REFERENCES}

[1] C. Berge, Espaces topologiques. Fonctions multivoques, Dunod, Paris, 1966. (in French).

[2] P. A. Bliman, LMI characterization of strong delay-independent stability of linear delay systems via quadratic lyapunov-krasovskii functionals, Syst. \& Contr. Lett., 43 (2001), pp. 263-274.

[3] J. Chen, G. Gu, And C. A. Nett, A new method for computing delay margins for stability of linear delay systems, Syst. \& Contr. Lett., 26 (1995), pp. 107-117.

[4] J. Chen and H. A. Latchman, Frequency sweeping tests for stability independent of delay, J. Math. Anal. Appl., 40 (1995), pp. 1640-1645.

[5] R. DAтKo, Remarks concerning the asymptotic stability and stabilization of linear delay differential equations, J. Math. Anal. Appl., 111 (1985), pp. 571-584.

[6] L. Dugard And E. Verriest, Stability and control of time-delay systems, vol. 228 of Lecture Notes in Control and Information Sciences, Springer-Verlag, 1998.

[7] K. Engelborghs, T. Luzyanina, and G. Samaey, DDE-BIFTOOL v. 2.00: a Matlab package for bifurcation analysis of delay differential equations, TW Report 330, Department of Computer Science, Katholieke Universiteit Leuven, Belgium, October 2001.

[8] K. Gu, V. L. Kharitonov, and J. Chen, Stability of time-delay systems, Contr. Eng., Birkhauser, Boston, 2003.

[9] K. Gu, S. I. Niculescu, AND J. Chen, On stability of crossing curves for general systems with two delays, J. Math. Anal. Appl., 311 (2005), pp. 231-253.

[10] J. Hale And S. Verduyn Lunel, Strong stabilization of neutral functional differential equations, IMA Journal of Mathematical Control and Information, 19 (2002), pp. 5-23.

[11] G. Hardy And E. Wright, An introduction to the theory of numbers, Oxford University Press, 1968.

[12] E. W. KAMEN, On the relationship between zero criteria for two-variable polynomials and asymptotic stability of delay differential equations, IEEE Trans. Automat. Contr., AC-25 (1980), pp. 983-984.

[13] - Linear systems with commensurate time delays: stability and stabilization independent of delay, IEEE Trans. Automat. Contr., AC-27 (1982), pp. 367-375.

[14] V. Kolmanovski and V. Nosov, Stability of functional differential equations, vol. 180 of Mathematics in Science and Engineering, Academic Press, 1986.

[15] J. Louisell, Absolute stability in linear delay-differential systems: Ill-possedness and robustness, IEEE Trans. Automat. Contr., 40 (1995), pp. 1288-1291.

[16] N. MacDonald, Two delays may not destabilize although either delay can, Math Biosciences, 82 (1986), pp. 127-140. 
[17] - An interference effect of independent delays, IEE Proc. Contr. Theory \& Appl., Pt. D, 134 (1987), pp. 38-42.

[18] W. Michiels, K. Engelborghs, D. Roose, and D. Dochain, Sensitivity to infinitesimal delays in neutral equations, SIAM Journal on Control and Optimization, 40 (2002), pp. 11341158.

[19] W. Michiels and T. Vyhlídal, An eigenvalue based approach to the stabilization of linear time-delay systems of neutral type, Automatica, 41 (2005), pp. 991-998.

[20] S.-I. Niculescu, Stability and hyperbolicity of linear systems with delayed state: A matrix pencil approach, IMA J. Math. Contr. Information, 15 (1998), pp. 331-347.

[21] - Delay effects on stability: A robust control approach, LNCIS, Springer-Verlag, Heidelberg, 2001.

$[22]-$ On interference phenomena in stability of linear systems with multiple delays, in Proceeding of 4th Asian Control Conference, Singapour, 2002.

[23] R. Sipahi And N. Olgac, Complete stability robustness of third-order lti multiple time-delay systems, Automatica, 41 (2005), pp. 1413-1422.

[24] O. TOKER AND H. ÖZBAY, Complexity issues in robust stability of linear delay-differential systems, Math., Contr., Signals, Syst., 9 (1996), pp. 386-400. 